\title{
An Unusually Aggressive Irritation Fibroma: A Diagnostic Dilemma
}

\author{
Ryan Yeo Zile Ware ${ }^{1}$, Yijin Jereme Gan ${ }^{1}$, Amit Anand Karandikar ${ }^{2}$, Yee Lin Tang ${ }^{3}$, Ming Yann Lim ${ }^{1}$ \\ Departments of ${ }^{1}$ Otorhinolaryngology, ${ }^{2}$ Diagnostic Radiology and ${ }^{3}$ Histopathology; Tan Tock Seng Hospital, Singapore.
}

\section{Corresponding Author:}

Dr Ming Yann Lim

Email: ming_yann_lim@ttsh.com.sg

This is an Open Access article distributed under the terms of the Creative Commons Attribution License (creativecommons.org/ licenses/by/3.0).

Received

Accepted

September 13, 2020

Published

April 21, 202

June 30, 2021

\begin{abstract}
Background: Irritation fibromas are benign tumors of connective tissue origin that occur in response to chronic irritation or trauma. Case Report: An 84-year-old lady presented with a large exophytic right maxillary gingival lesion with rapid progression over 3-months. Magnetic resonance imaging demonstrated a large heterogenous lobulated mass of the right maxillary alveolus measuring $4.3 \times 3.9 \times 4.3 \mathrm{~cm}$ with extension across the midline but no frank invasion.Histology from excision biopsy confirmed irritation fibroma. Conclusion: While classically benign and indolent, this unusual case illustrates the extraordinary size and aggressive clinical appearance they may demonstrate if left untreated. Awareness of such presentations is important as a differential of a large oral cavity mass.
\end{abstract}

Keywords: Biopsy, Female, Fibroma, Magnetic Resonance Imaging, Mouth.

\section{Introduction}

Benign fibrous growths arising from the soft tissues of the oral cavity are fairly common and encompass a broad range of pathologies. By far the most common of oral fibrous growths is the irritation fibroma - exophytic soft tissue lesions arising from the oral mucosa. These represent focal fibrous hyperplasia in response to local trauma or chronic irritation, rather than true benign neoplasms as their name implies. Irritation fibromas occur at sites subject to chronic irritation such as the plane of occlusion of the maxillary and mandibular teeth, the lips and lingual surface. They are classically described as well demarcated, slow growing lesions which rarely exceed $1-2 \mathrm{~cm}$ in size [1]. Our report presents a case of an unusually large gingival irritation fibroma, illustrating the extraordinary size and appearance they can demonstrate if left untreated, and the resultant difficulties in diagnosis when faced with such lesions.

\section{Case Report}

An 84-year-old Chinese lady, with a history of cognitive decline, was referred to the otorhinolaryngology clinic with a 3-month history of a progressively enlarging right maxillary gingival lesion. Examination revealed a large exophytic lesion arising from the right upper gingiva extending to the hard palate [Fig.1]. Flexible naso-endoscopy and the rest of the head and neck examination were unremarkable.

Incisional biopsy under local anesthesia was performed in the clinic and magnetic resonance imaging (MRI) was performed. Initial

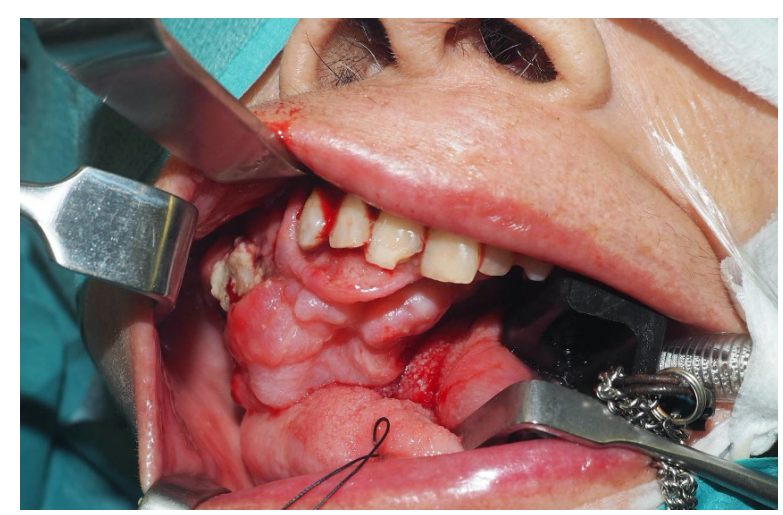

Fig.1: Large exophytic lesion arising from the right upper gingiva extending to the hard palate. 
histology revealed no overt malignancy within the sample; non-specific polypoid tissue with ulcerated mucosa and acute stromal inflammation was reported. MRI illustrated a large heterogeneously enhancing lobulated mass of the right maxillary alveolus and hard palate crossing the midline and measuring $4.3 \times 3.9 \times 4.3 \mathrm{~cm}$ without frank invasion of surrounding structures [Fig.2].To avoid missed malignancy due to sampling error during initial incision biopsy, wide excision of the mass was performed under general anesthesia for diagnostic confirmation. Intra-operatively, a large $6 \mathrm{~cm}$ mass was seen to arise from the gingiva over the right maxillary pre-molars with a loose tooth found within the mass and histological examination was performed.

Final histology showed a polypoid lesion with fibrovascular stroma covered by hyperplastic squamous epithelium, with an underlying proliferation of spindle to stellate-shaped cells loosely arranged in a myxoid to collagenous stroma [Fig.3]. This was suggestive of a large irritation fibroma.

\section{Discussion}

The oral cavity is frequently subject to chronic irritation and trauma that can result in reactive tumor-like non-neoplastic lesions. Such reactive lesions have been reported to make up to $44 \%$ of all lesions observed in the oral cavity [2]. These may pose a diagnostic challenge as the clinical appearance and presentation of reactive lesions can resemble that of more aggressive diseases.

Our case report illustrates the extraordinary size and appearance reactive lesions can demonstrate, which may confound or delay diagnosis. This difficulty is compounded in large lesions as incomplete incisional biopsy samples that demonstrate benign histopathology may not be representative of the entire lesion and may not sufficiently rule out malignant disease. Irritation fibromas are classically described as slow-

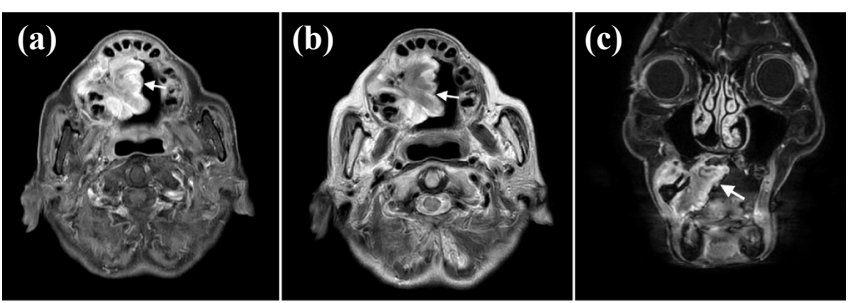

Fig.2(a): Axial post-contrast T1w fat-suppressed; (b): Axial T2w; (c): Coronal post-contrast T1w fat-suppressed. Image reveals a large polypoidal mass with lobulated outlines arising from the right maxillary alveolus protruding into the oral cavity. There is peripheral enhancement with central non-enhancing areas. The maxillary molar teeth are seen within the mass.

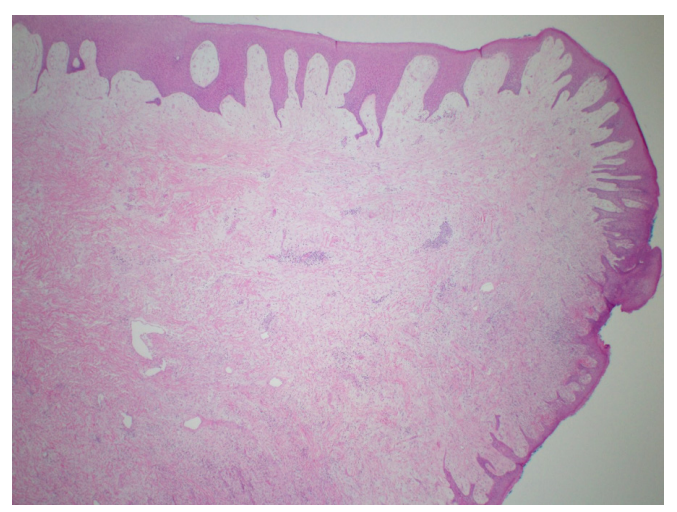

Fig.3: Scanning power image shows portion of a polypoid lesion with fibrovascular stroma covered with hyperplastic squamous epithelium. No odontogenic epithelial elements are seen in the deeper portions of the lesion $(H E \times 20)$.

growing, well-demarcated, sessile or pedunculated lesions which are firm to hard in consistency. The overlying mucosa is often normal in appearance but may demonstrate ulceration or hyperkeratosis following repeated trauma. Irritation fibromas rarely exceed $1.5 \mathrm{~cm}$ in size, although a few cases have been reported at 4-6 cm [3,4]. They occur most commonly at sites subject to chronic irritation such as the plane of occlusion of the maxillary and mandibular teeth, the lips and lingual surface. While irritation fibromas are often suspected clinically in small, indolent lesions with obvious sources of chronic irritation, differentials must be considered in atypical presentations. These include other reactive hyperplastic lesions, infection as well as benign and malignant neoplasms of the oral cavity. 
The most common reactive hyperplastic lesions of the oral cavity include the irritation fibroma, pyogenic granuloma, peripheral ossifying fibroma and peripheral giant cell granuloma. Differing incidence of the above lesions have been reported, with numerous studies reporting irritation fibromas as the most common reactive lesion (up to $57 \%$ ) [5-8]. Infective lesions of the oral cavity are reported to make up $30 \%$ of all oral lesions [3]. These are often of viral etiology, although bacterial infections and abscesses, fungal infections and tuberculous infections may also occur. Confirmation with cultures and RNA polymerase chain reaction (PCR) testing may be considered in patients with a high index of suspicion.

Benign tumors may arise from the various structures within the oral cavity of which the most common include the papilloma, granular cell tumor, neurofibroma, hemangioma, ameloblastoma and pleomorphic adenoma [10]. Lastly and most crucially, malignant neoplasms of the oral cavity must be ruled out during evaluation. Squamous cell carcinoma represents the large majority of lesions, with adenocarcinoma, verrucous carcinoma, minor salivary gland carcinoma, lymphoma and other mesenchymal malignancies accounting for the remainder $[2,10]$.

Diagnosis of irritation fibroma is confirmed on histologic examination which reveals an unencapsulated, nodular mass of dense fibrous connective tissue which may be hyalinized. Due to chronic irritation, mild inflammatory cell infiltrate may be present and surface epithelium may demonstrate hyperkeratosis, hyperplasia, atrophy and/or ulceration. In clinically indeterminate lesions, imaging may provide supplemental information. MRI shows delineation of soft tissue tumors and aids in determining involvement of adjacent structures. Reactive lesions typically appear as ill-marginated, well-enhancing, superficial soft tissue mass-like lesions, which are iso-intense on T1-weighted images and hyperintense on T2-weighted images; these findings may resemble those of mucosal carcinomas [11].
Treatment of reactive lesions is by conservative excision and recurrence is rare unless the precipitating chronic trauma continues. Awareness of this condition is important as a differential of a large oral cavity mass.

\section{Conclusion}

Reactive lesions are the most common tumorlike lesions of the oral cavity and are classically indolent, small and benign in appearance. Treatment is by conservative excision and removal of the offending source of chronic irritation. Some reactive lesions can mimic neoplastic disease and may be difficult to distinguish from malignant tumors both clinically and radiologically. Diagnosis in such lesions may only be clinched on complete excision and histopathological examination. It is important to note that clinical behavior of reactive lesions may vary in different populations, and awareness of atypical presentations and differential diagnoses is critical when faced with aggressive appearing disease.

Contributors: RWYZ: manuscript writing, patient management; LMY, GYJ: manuscript editing, patient management; KAA: critical inputs into the manuscript and imaging. TYL: intellectual input into the manuscript and histopathology. LMY will act as a study guarantor. All authors approved the final version of this manuscript and are responsible for all aspects of this study.

Funding: None; Competing interests: None stated.

\section{References}

1. Esmeili T, Lozada-Nur F, Epstein J. Common benign oral soft tissue masses. Dent Clin North Am. 2005;49:223240.

2. Weir JC, Davenport WD, Skinner RL. A diagnostic and epidemiologic survey of 15,783 oral lesions. J Am Dent Assoc. 1987;115:439-442.

3. Kinoshita H, Ogasawara T, Toya T, Makihara R, Hirai R, Kawahara E. Slow-growing large irritation fibroma of the anterior hard palate: A case report using immunohistochemical analysis. J Maxillofac Oral Surg. 2016;15(S2):253-257.

4. Lanjekar A, Kulkarni S, Akhade S, Sonule S, Rathod U. An unusually large irritation fibroma associated with gingiva of lower left posterior teeth region. Case Rep Dent. 2016;2016:1-4. 
5. Neville BW, Damm DD, Allen CA, Bouguot JE. Oral and Maxillofacial Pathology. $2^{\text {nd }}$ ed. Philadelphia: W.B. Saunders; 2002.

6. Reddy V, Saxena S, Saxena S, Reddy M. Reactive hyperplastic lesions of the oral cavity: A ten year observational study on North Indian population. J Clin Exp Dent. 2012;4:e136-140.

7. Eversole LR, Rovin S. Reactive lesions of the gingiva. J Oral Pathol. 1972;1:30-38.

8. Kfir Y, Buchner A, Hansen LS. Reactive lesions of the gingiva: A clinicopathological study of 741 cases. J Periodontol. 1980;51(11):655-661.

9. Kadeh H, Saravani S, Tajik M. Reactive hyperplastic lesions of the oral cavity. Iran J Otorhinolaryngol. 2015;27(2).

10. Flint PW. Cummings Otolaryngology: Head \& Neck Surgery. Philadelphia, PA: Elsevier/Saunders; 2015.

11. Kim ST, Kim HJ, Park IS, Park SW, Kim WH, Kim YM. Chronic, reactive conditions of the oral cavity simulating mucosal carcinomas. Clin Imaging. 2005;29(6):406-411. 